\title{
Viajeros interurbanos en un contexto patrimonial ferroviario: evaluación cuantitativa del servicio Regional Victoria - Temuco, Chile
}

\section{Intercity travelers in a railway heritage context: Quantitative assessment of the Victoria - Temuco Regional Service, Chile}

\author{
Hernán Riquelme Brevis ${ }^{1}$ \\ ORCID: https://orcid.org/0000-0002-9686-6284 \\ Correo electrónico: h.riquelmebrevis@gmail.com \\ Jorge Canales Urriola ${ }^{1}$ \\ ORCID: https://orcid.org/0000-0002-5594-221X \\ Javiera Azócar Weisser ${ }^{1}$ \\ ORCID: https://orcid.org/0000-0001-7849-4942 \\ Matías Riquelme Brevis ${ }^{2}$ \\ ORCID: https://orcid.org/0000-0003-1205-7082 \\ ${ }^{1}$ Universidad Autónoma de Chile, Temuco, Chile. \\ ${ }^{2}$ Universidad de la Frontera, Temuco, Chile.
}

\begin{abstract}
Este trabajo fue financiado por la Vicerrectoría de Investigación y Postgrado de la Universidad Autónoma de Chile, a través del proyecto DIUA 1702019. El autor principal, como miembro del claustro académico del Magíster en Gestión del Patrimonio y Turismo Sostenible (Universidad Autónoma de Chile), agradece los comentarios de los estudiantes de la asignatura Fundamentos Teóricos y Metodológicos para la Gestión del Patrimonio y

Turismo, que permitieron enriquecer la presente investigación.
\end{abstract}

\section{Resumen}

En términos epistemológicos, se considera el ferrocarril un objeto de estudio que permite observar tanto su rol vinculado exclusivamente al transporte como también el valor asignado a nivel simbólico y material por los habitantes de zonas urbanas y rurales de los territorios. Por este motivo, la presente investigación analiza la valoración que hacen los pasajeros del servicio ferroviario respecto a su impacto en el desarrollo de las actividades cotidianas. Además de la utilización de un enfoque metodológico cuantitativo, se estableció contacto con viajeros del ferrocarril $(n=224)$ para así analizar la apreciación del servicio mediante variables como funcionamiento ferroviario, motivaciones de uso y otros atributos valorativos. Dentro de los resultados, analizados en base a variables particulares, destaca una evaluación ciertamente positiva a nivel de efectividad de transporte, la necesidad de ampliar los servicios ofrecidos y un estrecho vínculo entre el ferrocarril y la cultura local e historia familiar, lo cual influye en la elección de este dispositivo de transporte por sobre otros.

Palabras clave

Patrimonio ferroviario, transporte, viaje interurbano.

\begin{abstract}
In epistemological terms, the railway is considered as a study object that allows multiple observations. On the one hand, its exclusive role is transporting, but on the other hand, its symbolic and material value for the inhabitants of urban and rural areas of the territories. This study analyzes the railway service valuation by its users regarding its significance in their daily activities. Using a quantitative methodological approach, railway passengers were contacted $(n=224)$ to analyze their assessment using variables, such as railway operation, the reason for using the service, and valuation characteristics. Among the findings, analyzed based on particular variables, there is a distinct positive assessment of the service efficacy, the need to widen the services offered, and a close bond between the railway and local culture and family history. This is one of the factors affecting this choice of transportation from others.
\end{abstract}

\section{Keywords}

Railway heritage, transportation, inter-city travel. 


\section{Introducción}

El origen del ferrocarril en Chile está estrechamente ligado a la relación entre producción económica y avances tecnológicos. En un primer momento, será la minería del norte del país, hacia mediados del siglo XIX, la industria que empujaría la construcción de las primeras líneas ferroviarias, para luego seguir con el sur agrícola. En efecto, el ferrocarril fue moldeado siguiendo las formas productivas de la agricultura y de la minería, siendo la presión exportadora la que impulsó las primeras iniciativas para mejorar los transportes y las comunicaciones (Guajardo, 2007).

La necesidad de transportar de manera expedita las ingentes cantidades de mineral, fue el hecho que desencadenó la construcción del primer ferrocarril en suelo nacional, conectando la ciudad de Copiapó con el puerto de Caldera a partir del año 1851. Así, "con esta vía férrea quedaba asentada en Chile una de las mayores empresas de la modernidad" (Alliende, 2017, p. 145).

El ejemplo del ferrocarril en el norte de Chile fue prontamente tomado en cuenta para ser replicado en otras regiones del país. De esta forma, a partir de mediados del siglo XIX, se discutiría la instalación de un tren entre Santiago y Valparaíso, iniciándose su construcción en 1855 (Alliende, 2017).

A partir de este ferrocarril, se pasó prontamente a planificar la extensión de la línea férrea hacia el sur del país, lo que significó que, hacia fines de la década de 1870, las líneas conectaran sin interrupciones desde Valparaíso hasta Angol (Alliende, 2017). Si bien los ferrocarriles del norte tuvieron un interés meramente económico, al ser una extensión de corporaciones mineras privadas, en el Valle Central y Sur de Chile el aporte del Estado en la instalación de la red ferroviaria dejó ver la importancia de fines políticos para la consolidación del territorio nacional (Alliende, 2017; Flores, 2012; Guajardo, 2007).

Tal como señala Flores (2012), la conformación del territorio nacional fue una de las principales tareas que emprendió el Estado chileno luego de obtener su independencia. La Guerra del Pacífico en el norte y la Ocupación de La Araucanía en el sur, son la prueba tangible de ello. Este último hecho se encuentra directamente relacionado con la construcción del ferrocarril en el territorio austral, en tanto se constituyó como una de las herramientas utilizadas por el Estado para apropiarse de este territorio "de frontera", introduciendo nuevas costumbres y tradiciones. De este modo:

el ferrocarril emerge como un dispositivo que permite conectar, vigilar, organizar y estructurar La Araucanía tanto en términos intrínsecos, desde la comunicación entre las principales ciudades de la región, como extrínsecos, con las regiones aledañas y la capital nacional. (Riquelme y Oyarce, 2019, p. 9)

Así, a partir de 1850 el Estado chileno centró su actuar en la zona sur con el fin de desunir el territorio mapuche -situado entre los ríos Biobío por el norte y Toltén por el sur- y rearticularlo bajo una nueva lógica territorial en la que la construcción del ferrocarril cumplió un papel central. La línea ferroviaria de La Araucanía no solo unió un territorio aislado, sino que permitió ampliar el poder y control del Estado chileno, fundando nuevas ciudades, producto del avance civilizador de los Estados-nación en territorios indígenas (Almandoz, 2013) ${ }^{1}$.

La Ocupación de La Araucanía, iniciada en 1861, finaliza oficialmente el 1 de enero de 1883, fecha en la cual la región comprendida entre el río Malleco, por el norte, y el

1 A partir la conquista, y en toda América Hispánica, fue parte central de la política de dominación territorial. Desde un inicio primó una planificación urbana que, siguiendo directrices precisas, se orientó a construir ciudades ordenadas (Guarda, 1978; Musset, 2009; Rojas-Mix, 2002). Las ciudades, pueblos y poblados fueron la estructura territorial base de toda la América española y un instrumento esencial de dominio (Dourton, 1994).

Si bien la formación de ciudades fue central durante la etapa de conquista española, en América Latina se observa un segundo momento fundacional coincidente con la construcción de los Estados-nación (Núñez, 2010; Sagredo, 2010). 
río Toltén, por el sur, sería incorporada a la República de Chile, formándose el llamado "Territorio de colonización". A partir de 1883 comienzan a llegar, desde el puerto de Talcahuano al nuevo territorio incorporado, colonos suizos, alemanes, belgas, franceses, rusos, ingleses, españoles e italianos (Alliende, 2017).

El hito central de la instalación del ferrocarril en este territorio lo constituye la edificación del viaducto del Malleco, puente ferroviario construido en Francia a partir de 1887 e inaugurado en La Araucanía en 1890, que terminaría de vincular el centro del país con la Región de la Frontera.

Gracias a este avance, en octubre de 1890 el ferrocarril hace su ingreso en la estación de Victoria, y en noviembre de 1892 el tren llega hasta Lautaro. El 31 de diciembre finaliza la construcción del riel que conecta con Temuco. Sin embargo, no sería sino hasta 1895 que se haría entrega de la línea completa Victoria-Lautaro-Temuco a la Empresa de Ferrocarriles del Estado, mientras que en 1898 se inauguró la línea Temuco-Pitrufquén (Alliende, 2017). En los años posteriores, el tren se siguió ampliando hacia el sur, anexando a la red la ciudad de Puerto Montt en 1913.

Durante gran parte del siglo XX, el ferrocarril fue el principal medio de transporte nacional. Tal como señala Guerrero (2017): "las diferentes regiones del país construyeron su identidad y su desarrollo económico y social en torno al ferrocarril" (p. 60), y fue uno de los inventos de la era industrial que más impacto causó, tanto en términos económicos como políticos. La forma del territorio chileno, una larga y angosta franja, facilitó el desarrollo y consolidación del tren durante más de un siglo. Al eje central (norte-sur) se le anexaban una serie de ramales (este-oeste) que conectaban el territorio nacional desde Arica hasta Puerto Montt.

Sin embargo, a mediados de los años ochenta "se inició un proceso de privatización que implicó el cierre de muchos ramales, especialmente los más pequeños y económicamente menos rentables" (Guerrero, 2017, p. 60). A partir de entonces, y de manera paulatina, el ferrocarril fue cayendo en un descuido y abandono progresivo. Entre las razones del fin del ferrocarril en Chile destaca la imposición de un nuevo sistema económico neoliberal, instaurado por la dictadura iniciada el 11 de septiembre de 1973.

Este brutal cambio implicó, entre otras cosas, el desmantelamiento del Estado de bienestar, que tuvo como resultado práctico la privatización de muchas de las empresas estatales garantes de derechos sociales (Guerrero, 2017). En el marco de este proceso, el año 1979 marca el fin del subsidio estatal entregado a la Empresa de Ferrocarriles del Estado (EFE), hecho que significó el deterioro de una de las empresas más importantes en la historia moderna del país.

A inicios de los 2000, se instala desde el gobierno de turno la idea de refundar la empresa para dar nueva vida al ferrocarril en el país. Es así como, bajo el gobierno de Ricardo Lagos (2000-2006), se establece el llamado Plan Trienal que pretendía reanudar el servicio de ferrocarriles desde Santiago hasta Puerto Montt.

Durante el año 2003, se reinició el servicio Santiago-Temuco (Terrasur), y en diciembre de 2005 se inauguró el tramo Victoria-Puerto Montt. Problemas de gestión, sumados a una falta de recursos en el área, imposibilitaron la continuación del servicio, que solo duró algunos años y que no fue continuado por el gobierno sucesor de Michelle Bachelet.

A pesar de los contratiempos y olvidos, desde hace una década se ha producido en Chile una revalorización social del ferrocarril, ligada a la importancia y profundización de la noción de patrimonio cultural, hecho que se ha manifestado en el surgimiento de diversas acciones de recuperación, preservación, gestión y puesta en valor del patrimonio ferroviario (Guerrero, 2017, p. 60).

Bajo este contexto es necesario destacar que "la historia de La Araucanía ha estado fuertemente influenciada por el papel del ferrocarril en las dinámicas territoriales, la construcción del paisaje cultural y las prácticas de movilidad desarrolladas por sus habitantes" (Riquelme 
y Oyarce 2019, p. 6). En la actualidad existe en la región un solo servicio ferroviario que conecta las ciudades de Temuco y Victoria, y que sin duda es parte de la identidad de la zona. Este servicio opera en 65 kilómetros de ruta, conectando las comunas de Victoria, Perquenco y Lautaro con la capital regional, Temuco.

Considerando esta problemática, la presente investigación se pregunta cómo los pasajeros construyen la valoración del servicio ferroviario en relación con variables motivacionales, patrimoniales y económicas. Por esta razón, el objetivo es identificar la valoración que efectúan los usuarios del ferrocarril Victoria-Temuco en aspectos relacionados con el funcionamiento y los motivos de su utilización, como también determinar variables de carácter económico y cultural que permiten considerar aspectos negativos y positivos del servicio, particularmente, en lo relativo a la comprensión del patrimonio.

La hipótesis planteada es que, a pesar de que el ferrocarril constituye un dispositivo de transporte fundamental para la historia de la región y el país, la disminución de sus recorridos impacta negativamente en la calidad de vida de las personas (especialmente habitantes de la ruralidad), que necesitan de este servicio. No obstante, existe una valoración positiva del ferrocarril como medio de transporte con alto arraigo en la cultura, política y economía de La Araucanía.

El artículo incluye cinco secciones, además de la introducción. En la primera parte se desarrolla una discusión teórica que permite enfocar la investigación en base a conceptos como patrimonio ferroviario, memoria e identidad. En la segunda sección, se presenta el diseño metodológico, explicando los criterios de la muestra, técnica de producción de información y aspectos éticos. Posteriormente, se presentan los resultados del estudio en base a tres variables, a saber: funcionamiento ferroviario, motivaciones de uso y aspectos valorativos. En el cuarto apartado, se desarrolla una discusión entre los resultados y la teoría. Finalmente, hay que destacar que el presente trabajo permite nutrir la producción científica sobre el patrimonio ferroviario en un territorio que se desarrolló en directa relación con la construcción y desplazamiento de los ferrocarriles, aspecto que se aborda en las conclusiones del estudio.

\section{Discusión teórica}

Existe una relación fundamental entre el ferrocarril y el patrimonio material (desde la infraestructura) e inmaterial (desde los sujetos que revitalizan los objetos inanimados). Esta realidad permite observar dinámicas relacionadas con la identidad, patrimonio y relaciones de poder, lo cual será analizado en esta sección a través de aproximaciones teórico-conceptuales.

En su concepción más tradicional, el patrimonio ferroviario se sitúa en la categoría de patrimonio material industrial, y se entiende como los bienes heredados de las explotaciones ferroviarias (Méndez y Cuéllar, 2017). Generalmente, estos bienes se asocian a una materialidad que consta, principalmente, de locomotoras, vagones, líneas férreas, estaciones y maestranzas, los que forman parte del paisaje, comprendido desde la acción y creación humana con la naturaleza (Valencia, 2017).

Sin embargo, al menos conceptualmente, progresivamente se ha ido considerando la dimensión inmaterial de este patrimonio. Méndez y Cuéllar (2017) definen el patrimonio ferroviario como el "conjunto de bienes materiales e inmateriales que articulan un complejo sistema de transporte que interacciona transversalmente con el medio natural, social y cultural y que genera una fuerte atracción social” (p. 278). Complementariamente, Torres (2013) se refiere a las maestranzas como conjuntos arquitectónicos que reflejan un contexto histórico y un sistema industrial particular, pero que además forman parte de la memoria y la identidad de un territorio.

Estas conceptualizaciones interpretan las definiciones más amplias de patrimonio cultural, las que, sin negar la materialidad de este, ponen el énfasis en su uso social, el que va cambiando en el tiempo de acuerdo con los intereses de los sujetos que lo utilizan, renovando parte de sus elementos significativos y dejando otros en desuso, lo que transforma la fisonomía del patrimonio mismo (Prats, 1997). En otras palabras, el patrimonio 
representa formas de concebir y vivir el mundo, y su relevancia no se halla tanto en su autenticidad como en su verosimilitud histórica, pues esta incluye los significados que los actores le asignan y las particulares formas en que relacionan sus elementos constitutivos (García Canclini, 1999).

El patrimonio cultural e histórico se "construye" desde el presente y a partir de las necesidades de las sociedades actuales. Esto implica que el patrimonio supone transformaciones en sus contenidos, pues lo coyuntural genera reinterpretaciones, nuevas valoraciones y cambios en la selección de sus elementos significativos, lo que repercute en su transmisión, difusión y apropiación (Rodríguez, 1999).

De aquí que el patrimonio no deba entenderse únicamente como el objeto patrimonial, sino como el proceso de construcción de una significación simbólica sobre ese objeto que existe solo cuando se enuncia, pero las enunciaciones sobre un objeto patrimonial no son idénticas ni necesariamente convergentes, lo que implica que exista un conjunto de significados diferentes, con orígenes diversos, que actúan como capas significativas que se van superponiendo en el tiempo y en los que se involucra el imaginario de los sujetos que lo interpretan (Dormaels, 2011).

Al considerar la diversidad de significaciones sobre los objetos patrimoniales, aparece también cierta conflictividad entre los sujetos que los significan. García Canclini (1999) señala que los bienes patrimoniales no pertenecen a todos y que los grupos sociales se apropian, de acuerdo con su capital cultural, de forma diferente y desigual de la herencia cultural. Así, el patrimonio constituiría un espacio de lucha material y simbólica usada por la hegemonía cultural para reproducir diferencias sociales. De aquí la relevancia que ha adquirido el patrimonio local y popular como herramienta de los grupos subalternos para afirmarse como sujetos sociales, reforzando sus propias memorias e identidades.

El patrimonio viene a desempeñar un rol importante en la dimensión simbólica de la identidad, pues logra movilizar significaciones que son compartidas por uno o varios grupos, fortaleciendo sus procesos de identificación. Pero la identidad no es solo producto del patrimonio, pues paralelamente activa la conciencia patrimonial de un grupo, implicando un proceso dialéctico en el que las interacciones identitarias constituyen el centro de la producción simbólica del patrimonio, movidas por la búsqueda de elementos de identidad en la propia memoria (Rojas, 2015).

Guerrero (2017), refiriéndose al ferrocarril del sur de Chile, identifica tres momentos. En primer lugar, la memoria de los ex ferroviarios, memoria dolida y de resistencia, algo inmóvil y crítica respecto al desmantelamiento de ferrocarriles, señalando este proceso como un quiebre de la memoria. En segundo lugar, la memoria de exusuarios y amantes de los trenes, nostálgica pero activa, que articula acciones de rescate y conservación del patrimonio ferroviario material. En tercer lugar, la memoria oficial, aquella del Estado y la empresa de ferrocarriles, que es objetual, anecdótica y orientada al turismo, cuyas acciones no contribuyen a la transmisión y preservación de memorias ferroviarias, sino a partir de una idea desterritorializada y cosificada del patrimonio, desentendiéndose de la memoria de las comunidades (Guerrero, 2012, 2017).

Como señala Jelin (2002), la memoria se sitúa en el "espacio de experiencia” del presente. Tanto ese presente como las expectativas de futuro contribuyen a forjar la memoria, la que se nutre, además, de las experiencias y expectativas de otros, adquiriendo así una multiplicidad de tiempos y sentidos. De esta forma, la memoria colectiva sería un entretejido de "memorias compartidas, superpuestas, producto de interacciones múltiples, encuadradas en marcos sociales y en relaciones de poder" (Jelin, 2002, p. 22).

Estas memorias buscan fijar parámetros de identidad, seleccionando ciertos "recuerdos", mientras que esos parámetros se convierten en marcos sociales para encuadrar la memoria. En consecuencia, memoria e identidad se constituyen de forma recíproca (Jelin, 2002). 
Esta relación entre identidad y memoria es precisamente lo que concierne al patrimonio, razón por la cual éste ha adquirido mayor interés para los actores sociales de hoy. Según Rojas (2015), la modernidad globalizada ha provocado ciertas ansias de pasado, por lo que gran parte de la ciudadanía ha recomenzado a valorar su pasado, a recuperar su memoria y a constituir una audiencia favorable al patrimonio, dirigiendo estas acciones hacia la búsqueda de espacios de identidad.

En este contexto, las disputas por la memoria y la identidad repercuten en el patrimonio, convirtiéndolo en un campo de confrontación simbólica inevitable en cuanto instrumento para la representación de una identidad (Prats, 1997). Al mismo tiempo, la confrontación entre las distintas visiones del patrimonio está mediada por dinámicas de poder en la que los sectores dominantes buscan instalar un patrimonio hegemónico con el fin de homogeneizar la cultura y las identidades.

Sin embargo, la emergencia de patrimonios informales, deudores de una identidad comunitaria no formalizada por el poder, presenta dinámicas de construcción patrimonial desde abajo (Guerrero, 2017). Este patrimonio autorreconocido - público popular - se opone al patrimonio oficial -público aristocrático-, generando una dialéctica de conflictividad que se manifiesta en un "juego de intereses que devela la negatividad” o exclusión de ciertos referentes patrimoniales (Cantar, 2019, p. 6).

De esta forma, tanto la activación de referentes patrimoniales como la asignación de significados al patrimonio se sitúan en un escenario en que, variablemente, las lógicas hegemónicas y las contrahegemónicas se disputan el control significativo del patrimonio, produciendo un proceso desde el cual los individuos se reapropian subjetivamente de los sentidos de los bienes patrimonializados.

En otro orden de cosas, dirá Augé (2009) que un viajero puede reconocerse por la forma de moverse, su apariencia o su modo de proceder, lo que se puede percibir con ojo antropológico en los viajes interurbanos, donde personas de distintas latitudes convergen en un espacio-tiempo. En este sentido, viajar constituye una práctica que involucra una planificación que trasciende el tiempo exclusivamente ligado al desplazamiento, donde el tiempo-espacio de planificación del viaje como también el arribo, son forma y parte de la movilidad (Gutiérrez, 2012).

Asimismo, García (1994) plantea que viajar se torna una actividad constitutiva del ser humano, donde aquel que se mueve de un lugar a otro conlleva plantear qué motivaciones impulsan el viaje. Al respecto, las ciencias sociales han dado múltiples lecturas al viajar, como fenómeno central de la historia social, donde categorías como trabajo, turismo, salud o vivienda abren múltiples entradas analíticas para comprender el viaje como actividad esencial de los modos de vida contemporáneos.

Es interesante ver cómo el patrimonio ferroviario se nutre tanto de su propia historia, ligada íntimamente al territorio por el que transita y une, como de la realidad presente en el día a día del uso de este servicio. En ese sentido, el ferrocarril Victoria-Temuco no es solo un objeto patrimonial estanco para ser exhibido en un museo, sino que cumple funciones cotidianas de construcción de una historia colectiva, mostrando de manera clara la relación entre memoria, identidad y patrimonio.

\section{Diseño metodológico}

\section{Tipo de estudio}

La Araucanía es una de las 16 regiones que conforman el territorio nacional. Posee 32 comunas, la ciudad de Temuco es su capital. Según el Censo (INE, 2017), su población es de 957.224 habitantes, donde un 71\% reside en zonas urbanas y un $29 \%$ en zonas rurales. Respecto a variables socioeconómicas, cabe señalar que posee un $28,5 \%$ de su población en situación de pobreza multidimensional (Ministerio de Desarrollo Social, 2017), dato que la posiciona en el nivel más alto de pobreza en el territorio nacional.

A partir de un enfoque metodológico cuantitativo, transversal y exploratorio, la recolección de información se efectuó en la Región de La Araucanía durante las temporadas de invierno y primavera del año 2019, 


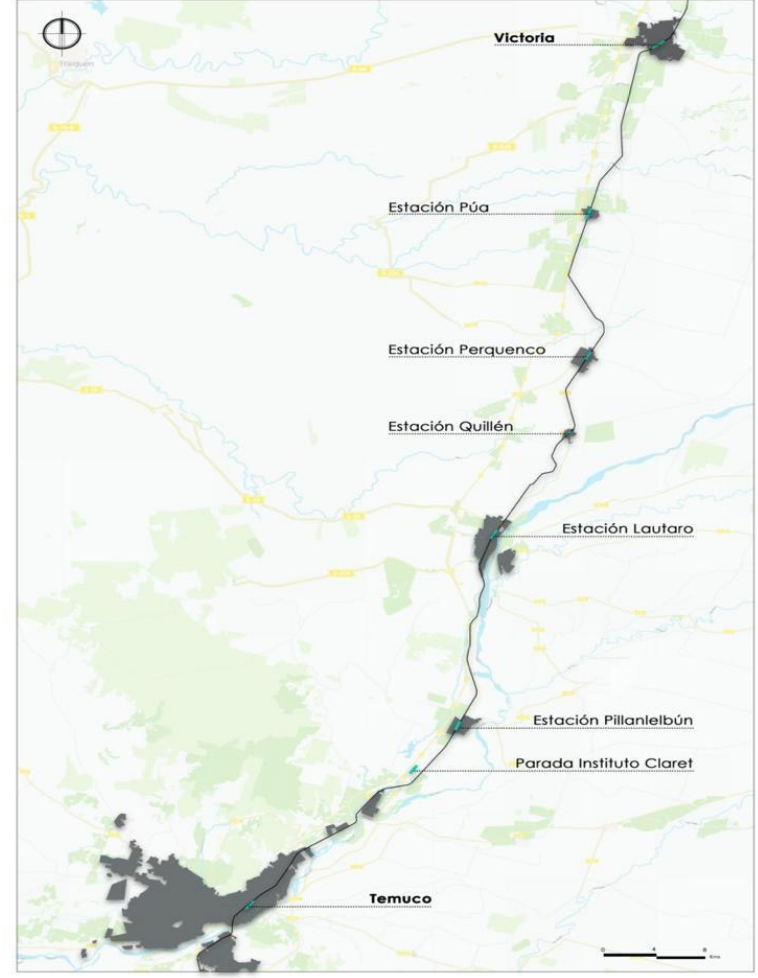

Fuente: Elaboración propia.

específicamente, en los tiempos de viaje de los pasajeros del servicio ferroviario regional Victoria-Temuco (Figura 1), el cual contempla las ciudades de Lautaro, Perquenco, Temuco y Victoria, y localidades como Púa y Pillanlelbún. Adicionalmente, ofrece un servicio exclusivo a los estudiantes del Instituto Claret, colegio ubicado en la zona norte de Temuco.

Respecto al cuestionario, confeccionado por profesionales de las ciencias sociales, cabe señalar que fue diseñado para aplicarse en tiempo de viaje, por lo que su extensión fue acotada con el objetivo de no interferir en demasía en los tiempos de desplazamientos de los viajeros. Contuvo 20 preguntas, y su estructura correspondió a secciones de carácter dicotómico, escala de Likert y abiertas breve.

Respecto a la secuencia de la recolección de información, en primer lugar, se instruyó a un equipo de encuestadores, formado por Estudiantes de pregrado de la Facultad de Ciencias Sociales y Humanidades de la universidad donde se desempeñan los investigadores responsables del proyecto, acudir a las estaciones del ferrocarril para recolectar información, para posteriormente, aplicar el cuestionario a viajeros. Finalmente, se procedió a desarrollar un análisis descriptivo, correlacional y de tablas dinámicas a través del programa SPSS.

\section{Muestra}

Luego de diseñar la encuesta, se aplicó el instrumento a una muestra de 224 personas, con un nivel de confianza de $90 \%$. Las características centrales de los participantes de la investigación (Tabla 1) se establecieron a partir de los siguientes criterios:

- Que al momento de responder el cuestionario residiera en la Región de La Araucanía.

- Que utilizara el ferrocarril como dispositivo de transporte fundamental para desplazarse a lugares vinculados al espacio laboral, educacional, como también actividades vinculadas a la realización de trámites personales o actividades turísticas, al menos una vez a la semana en días laborales, como también en temporadas estivales.

- Que fuera usuario del ferrocarril con vigencia de un año o más.

Tabla 1

Características centrales de los participantes de la investigación

\begin{tabular}{|c|c|}
\hline $\begin{array}{l}\text { Aspectos sociodemográficos de } \\
\text { la muestra }\end{array}$ & Porcentajes de respuestas \\
\hline Comuna de residencia & $\begin{array}{l}\text { Lautaro } 34 \% \text {, Padre Las Casas } \\
2 \% \text {, Perquenco } 6 \% \text {, Pillanlelbún } \\
3 \% \text {, Púa } 10 \% \text {, Temuco } 16 \% \text { y } \\
\text { Victoria } 29 \%\end{array}$ \\
\hline Sexo de participantes & $49 \%$ hombres y $51 \%$ mujeres \\
\hline $\begin{array}{l}\text { Motivo de utilización del } \\
\text { ferrocarril }\end{array}$ & $\begin{array}{l}\text { Trabajo 55\%, Estudio 34\%, } \\
\text { Realización de trámites } \\
\text { personales } 8 \% \text { y Turismo 3\% }\end{array}$ \\
\hline Edad de participantes & $\begin{array}{l}\text { 39,13 años. Edad mínima } 18 \text { años } \\
\text { y edad máxima } 86 \text { años. }\end{array}$ \\
\hline
\end{tabular}

Fuente: Elaboración propia.

\section{Técnica de producción de información}

El instrumento se elaboró con la finalidad de conocer la valoración del servicio de ferrocarriles a partir de 
Figura 2

Pasajeros en movimiento.
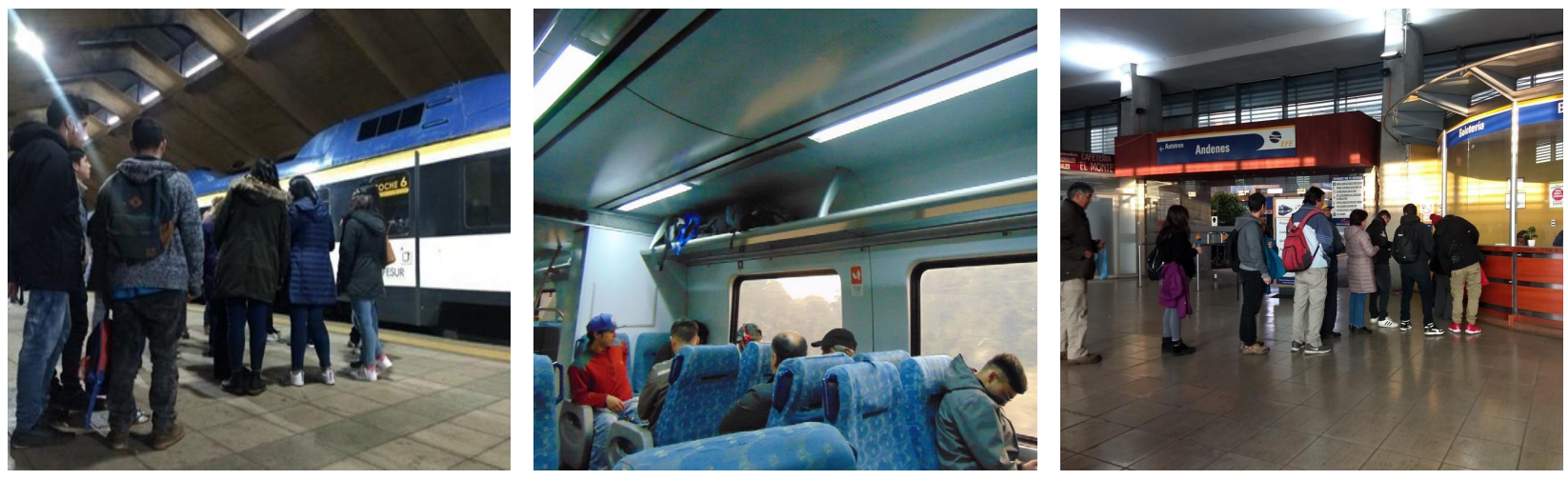

Fuente: Archivo de los autores.

la opinión de usuarios que de manera frecuente se trasladan en este medio de transporte (Figura 2). Se obtuvo información relativa a tres dimensiones de análisis, además de lo vinculado a aspectos sociodemográficos, que son detalladas en la Tabla 2.

Tabla 2

Dimensiones de análisis

\begin{tabular}{ll} 
Dimensiones de análisis & Definición operacional \\
\hline Funcionamiento ferroviario: & $\begin{array}{l}\text { Características a nivel funcional } \\
\text { del ferrocarril que permiten } \\
\text { considerar el rol social que } \\
\text { ocupa en ámbitos vinculados a } \\
\text { su relevancia sociocultural, nivel } \\
\text { de movilidad y operatividad. }\end{array}$ \\
\hline Factores de carácter económico, \\
cultural y social que implican \\
que los usuarios escojan el \\
servicio ferroviario por sobre \\
otros medios de transporte \\
disponibles.
\end{tabular}

Fuente: Elaboración propia.

\section{Aspectos éticos}

En primer lugar, se procedió a explicar brevemente los objetivos de la investigación a cada colaborador(a) antes de aplicar el instrumento, esto, con el objetivo de identificar su nivel de disposición a contestar las preguntas del instrumento. En segundo lugar, y una vez que hubiesen demostrado interés por participar en la investigación, se procedió a aclarar que tanto la información personal como los datos proporcionados serían tratados de manera anónima. Toda información proporcionada no sería usada en su contra y podrían dejar de responder el cuestionario cuando lo estimasen pertinente. Por último, hay que mencionar que no hubo ningún tipo de retribución económica, y que uno de los incentivos centrales de su participación residió en su contribución respecto a identificar variables que inciden en el nivel de valoración actual que tienen los usuarios respecto al ferrocarril como dispositivo de transporte con características patrimoniales en la región.

\section{Resultados}

\section{Funcionamiento ferroviario}

Con el objetivo de conocer la calificación que hacen los usuarios del ferrocarril respecto al servicio ofrecido por la empresa, la Figura 3 exhibe tres opciones que permiten considerar una mayor satisfacción en términos generales.

Figura 3

Calificación del servicio.

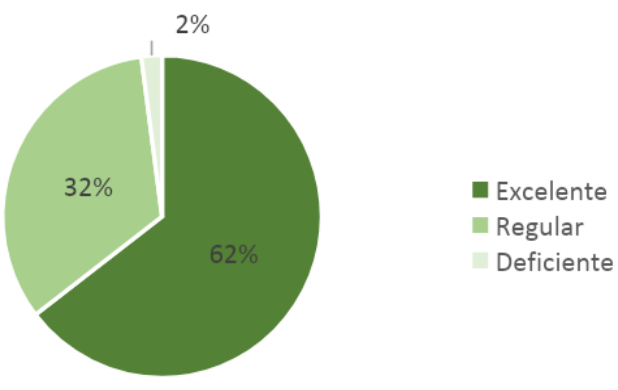

Fuente: Elaboración propia. 
Por un lado, queda en evidencia el predominio de la opción "excelente" con un $62 \%$ de las preferencias, lo cual se asocia a la capacidad de identificar un progreso en el servicio en relación con lo que anteriormente se ofrecía. En este sentido, el valor "excelente" se asocia a variables identificadas en los resultados, destacando: buen trato de los funcionarios hacia los pasajeros, infraestructura de viaje cómoda (resaltando asientos, calefacción y baños) y valor del pasaje (subsidiado) el cual alcanza una tarifa de $\$ 1.700$ (ida y vuelta) para el tramo Victoria-Temuco.

Por otro lado, emergió la opción "regular" con un 32\% de preferencias, cuya elección se relaciona con aspectos vinculados a un estancamiento del servicio en relación con la potencialidad que posee, es decir, nula capacidad de extensión de su ruta, fallas mecánicas, que en algunas ocasiones han ocasionado la supresión del servicio, y la poca capacidad de aumentar la velocidad (para un viaje de 65 kilómetros tarda una hora y treinta minutos). Finalmente, la opción "deficiente", con un $2 \%$ del total, está asociada a una valoración negativa en términos de infraestructura, atención al viajero y tiempos de viaje.

La Tabla 3, construida en base a las respuestas pesquisadas a partir de interrogantes dicotómicas, entrega relevante información respecto a valoraciones que atañen directamente a las necesidades de las comunas de la región respecto al funcionamiento del ferrocarril. En este sentido, el ferrocarril se torna un dispositivo de transporte fundamental para el desarrollo de las actividades cotidianas.

Queda en evidencia una mayor demanda por amplitud de servicios ofrecidos, ya que en la actualidad opera con tres servicios diarios de Victoria a Temuco, lo cual está estrechamente vinculado a una demanda planteada por los habitantes de territorios urbanos, específicamente habitantes de Temuco y Lautaro. Un factor de esta solicitud se relaciona con que los horarios del servicio están diseñados para que habitantes de las pequeñas comunas se desplacen a la capital regional. Muestra de lo mencionado es que el primer servicio diario comienza su viaje en la estación de Victoria a las 06:30, para llegar
Tabla 3

\begin{tabular}{lll} 
Euncionamiento del ferrocarril. & \multicolumn{1}{c}{$\begin{array}{l}\text { Porcentaje } \\
\text { opción Sí }\end{array}$} & $\begin{array}{l}\text { Porcentaje } \\
\text { opción No }\end{array}$ \\
\hline $\begin{array}{l}\text { Importancia para el transporte de } \\
\text { habitantes de la región. }\end{array}$ & $85 \%$ & $15 \%$ \\
\hline $\begin{array}{l}\text { Visibilidad de la relevancia social } \\
\text { del ferrocarril con el transcurso } \\
\text { del tiempo. }\end{array}$ & $9 \%$ & $91 \%$ \\
\hline $\begin{array}{l}\text { Amplitud de rango horario del } \\
\text { servicio ferroviario. }\end{array}$ & $84 \%$ & $16 \%$ \\
\hline
\end{tabular}

Fuente: Elaboración propia.

a Temuco a las 08:00, mientras que el primer servicio de Temuco a Victoria es a las 09:30, llegando poco después de las 11:00 a Victoria.

En una línea similar, los participantes de la investigación creen que, a medida que el tiempo pasa, el ferrocarril no adquiere mayor visibilidad y relevancia, lo cual impacta negativamente en las condiciones de viaje de la población. Los participantes atribuyen esta poca visibilidad de la relevancia del ferrocarril a las autoridades políticas (un $64 \%$ de la muestra genera esta asociación) y su poco compromiso con el desarrollo de la región.

\section{Motivaciones de uso}

En relación con las causas que implican la utilización del ferrocarril, cabe señalar que la muestra, tal como se mencionó anteriormente, lo utiliza, principalmente, para actividades relacionadas con el desplazamiento al lugar de trabajo, lugar de estudios, lugares de realización de trámites personales y desarrollo de prácticas turísticas. Estas actividades no son excluyentes entre sí.

En relación con aquellos enunciados que evocan la memoria y la relevancia del ferrocarril en la vida íntima de las personas y la región (enunciados 3, 4 y 5 de la Tabla 4), un $39 \%$ del total está muy de acuerdo y un $38 \%$ de acuerdo, con que el ferrocarril evoca la historia familia y la ciudad de residencia, sumando un $77 \%$ del total de preferencias. Esta realidad está íntimamente ligada con la relevancia del ferrocarril en la vida diaria de las ciudades, como también con el nexo que tiene 
Tabla 4

Implicancias en la utilización del ferrocarril.

\begin{tabular}{|c|c|c|c|c|c|}
\hline & $\begin{array}{l}\text { Muy en } \\
\text { Desacuerdo }\end{array}$ & En desacuerdo & Indeciso & De acuerdo & Muy de acuerdo \\
\hline $\begin{array}{l}\text { 1. En mis últimas vacaciones, utilicé el } \\
\text { ferrocarril con fines turísticos }\end{array}$ & $0 \%$ & $4 \%$ & $8 \%$ & $44 \%$ & $44 \%$ \\
\hline $\begin{array}{l}\text { 2. Durante el último tiempo, empleo el } \\
\text { ferrocarril diariamente para desarrollar mis } \\
\text { actividades }\end{array}$ & $3 \%$ & $13 \%$ & $22 \%$ & $32 \%$ & $30 \%$ \\
\hline $\begin{array}{l}\text { 3. En mi familia, el ferrocarril es utilizado por } \\
\text { más de un integrante }\end{array}$ & $11 \%$ & $26 \%$ & $17 \%$ & $31 \%$ & $15 \%$ \\
\hline $\begin{array}{l}\text { 4. Cuando utilizo el ferrocarril, generalmente, } \\
\text { recuerdo la historia de mi familia y mi ciudad }\end{array}$ & $3 \%$ & $10 \%$ & $10 \%$ & $38 \%$ & $39 \%$ \\
\hline $\begin{array}{l}\text { 5.Para el desarrollo de la región, creo que se } \\
\text { debiese revitalizar el ferrocarril }\end{array}$ & $0 \%$ & $7 \%$ & $7 \%$ & $34 \%$ & $52 \%$ \\
\hline
\end{tabular}

Fuente: Elaboración propia.

el ferrocarril con la vida laboral de las familias de la región, ya que décadas anteriores la empresa de ferrocarriles empleaba a una mayor cantidad de personas, construía vida social desde las estaciones e instauró costumbres y tradiciones en la región (Riquelme y Oyarce, 2019).

Respecto a la utilización del ferrocarril para fines turísticos (enunciado 1), cabe señalar que un $44 \%$ estuvo muy de acuerdo con este enunciado. Una de las posibles explicaciones a esta tasa de respuestas se origina en la potencialidad del ferrocarril como dispositivo que se vincula a la historia de los territorios, como también aspectos de carácter patrimonial material e inmaterial, y el paisaje rural y urbano, comprendiendo este último como el conjunto de lugares donde se desarrolla vida cotidiana (Ceniceros y Ettinger, 2020). La ubicación estratégica de las estaciones, en cada una de las ciudades, implica un circuito de diversos lugares que trascienden el viaje en sí, para transformarse en una experiencia de recreación y esparcimiento.

Finalmente, destaca la utilización del ferrocarril para el cumplimiento de las actividades diarias en días laborales (enunciado 2), registrando un alto nivel de conformidad, recalcando la opción "de acuerdo" con un $32 \%$ y "muy de acuerdo" con un $30 \%$.

Relacionado con el último enunciado de la Tabla 4, con el objetivo de identificar la frecuencia de utilización del servicio ferroviario en días laborales (lunes a viernes), la Figura 4 presenta la frecuencia por comunas. Para ello, se efectuó un análisis del total de encuestados por
Figura 4

Frecuencia de utilización de servicio de transporte en días laborales por comuna.

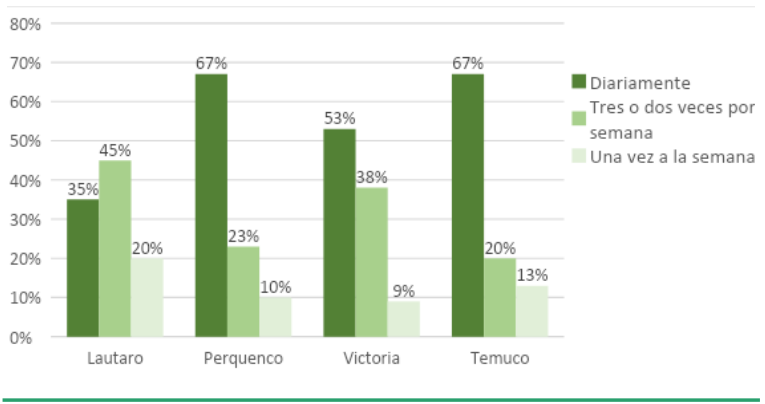

Fuente: Elaboración propia.

comunas $^{2}$ en relación con el nivel de periodicidad de viajes efectuados.

En primer lugar, la opción "diariamente" se impone en las cuatro comunas, destacando las comunas de Perquenco y Temuco con un $67 \%$ respectivamente. En segundo lugar, la alternativa "tres o dos veces por semana" muestra mayores resultados en las comunas de Lautaro y Victoria, con un $45 \%$ y $38 \%$ respectivamente. Finalmente, la opción "una vez a la semana" en la totalidad de los casos no supera el $20 \%$, se acentúa esta preferencia en Lautaro (20\%) y Temuco (13\%).

Una de las posibles causas de estos resultados se relaciona con los criterios de selección de la muestra, no obstante, llama la atención que a pesar de existir un horario de servicio que no resulta cómodo para los temuquenses, estos sean los que más utilizan el servicio. Los fundamentos de esta realidad pueden deberse a una mayor densidad

2 La localidad de Púa fue considerada en Victoria, mientras que Pillanlelbún en Lautaro. 
poblacional, como también a la realización de viajes cortos que no implican los dos extremos de la ruta del ferrocarril. En este sentido, el viaje en ferrocarril constituye una experiencia multifactorial, donde cada práctica implica situar la historia real de los territorios (Fusté-Forné, 2018).

\section{Atributos valorativos del servicio}

Con la finalidad de identificar acciones que permitan mejorar el servicio ferroviario, la Figura 5 presenta tres acciones prioritarias y reconocidas por los participantes. En primer lugar, y relacionado con la amplitud de rango horario observado en la Tabla 3, emerge la acción "ampliar el recorrido" con un 60\% de preferencias. Si en la actualidad el servicio involucra cuatro comunas, los participantes consideran que incluir nuevas comunas y regiones aledañas permitirá mayor visibilidad de sus lugares de origen y mayor conectividad con el resto del país. Esto es analizado de manera estratégica, como una oportunidad para mejorar la calidad de vida desde el servicio de ferrocarriles.

En segundo lugar, se posicionan las acciones "mejorar el horario" y "flexibilizar el servicio" con un 20\% de preferencias respectivamente. Cabe aclarar que flexibilizar el servicio implica más factores que los exclusivamente vinculados a los horarios de salida

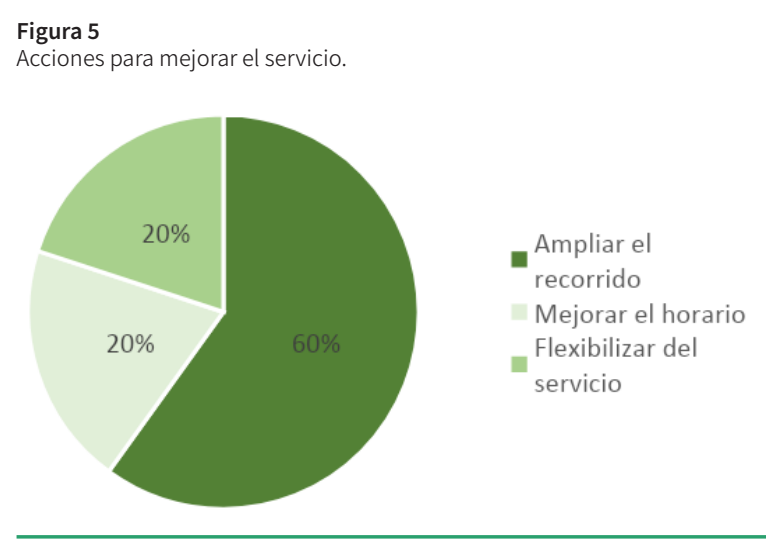

Fuente: Elaboración propia.

y llegada del ferrocarril, por ejemplo, paradas más extensas en las estaciones (13\%), venta de comida al interior del ferrocarril (7\%), destinos limitados (6\%), entre otras.

Respecto a las fortalezas y debilidades detectadas por los participantes de la investigación, la Figura 6 exhibe tres variables para cada opción. En el lado de las fortalezas, se repite la constante de vincularlo a factores económicos (48\%), debido al costo de sus pasajes que llega a ser muy inferior a otros medios de transporte público. En segundo lugar, se encuentra la variable "comodidad" (44\%), debido a una infraestructura que permite viajes confortables,
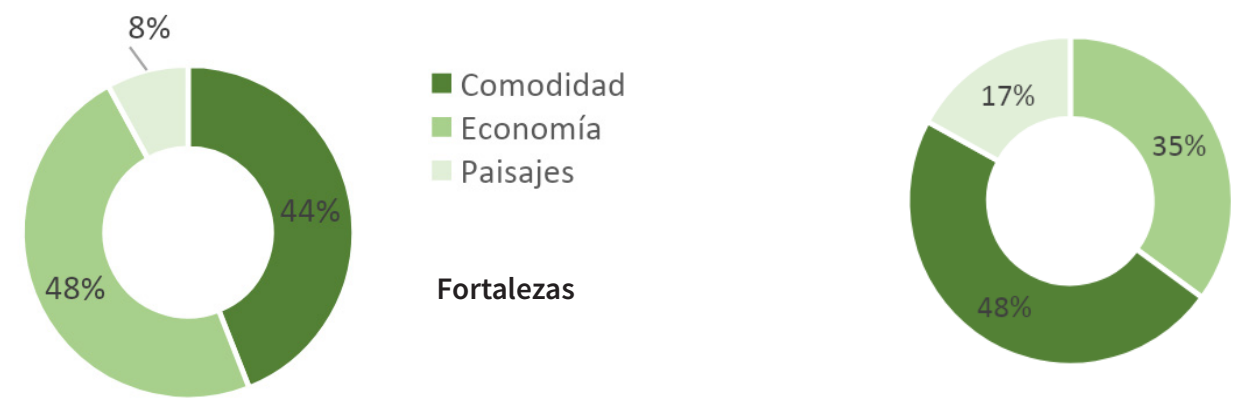

Horario

Destinos

Infraestructura

Debilidades

Fuente: Elaboración propia. 
haciendo del viaje en ferrocarril un momento atípico de las lógicas del transporte convencional (buses interurbanos), que generalmente implican menor comodidad. Por último, la variable "paisajes" (8\%) está relacionada con la propia infraestructura ferroviaria (puentes, estaciones, rieles, durmientes y paraderos), la geografía de la región (ríos, bosques y cordillera) y, finalmente, las transiciones entre lo urbano y lo rural.

El ferrocarril permite observar las ciudades desde una perspectiva más íntima, donde emergen patios de casas, zonas periféricas de las ciudades, lugares históricos y construcciones de alta relevancia arquitectónica y patrimonial, por ejemplo, edificio Marsano, Liceo Jorge Teillier, puente Quino, puente El Salto, taller ferroviario Victoria, entre otros.

Respecto a las debilidades, convergen tres variables que ya fueron observadas en los análisis anteriores. Los destinos (48\%), identificados como limitados, son apreciados como necesidad, pero en un estado de suspensión que inhibe la conectividad. Posteriormente, la variable "horario" (35\%), constituye un factor clave en la valoración del servicio, ya que es considerada como problemática para realizar las actividades diarias. Paradójicamente, la variable "infraestructura" (17\%), es observada como una debilidad. A pesar de ser identificada como un factor positivo en figuras anteriormente analizadas, en la generalidad, la infraestructura se instaura como el tercer factor débil del servicio de ferrocarriles.

Con el objetivo de identificar las variables que implican la globalidad del concepto de patrimonio ferroviario, los encuestados identificaron seis variables que operan como indicadores de esta dimensión, a saber: "estaciones" (38\%), como elemento icónico del patrimonio ferroviario material; "paisajes" (23\%), representado en la experiencia de viaje y las transiciones territoriales; "familia" (15\%), vinculado a la historia de vida personal y su relación con oficios y profesiones de familiares cercanos; "ciudades" (11\%) relacionado con el lugar habitado y las prácticas cotidianas concernientes con el papel del ferrocarril; "publicidad" (8\%), ligado a las campañas publicitarias relacionadas con la revitalización de los ferrocarriles en Chile, y, "recuerdos" (5\%), unido al sentimiento de añoranza por aquellos tiempos donde el ferrocarril cumplía un rol clave en la memoria, la vida social y la planificación de las ciudades.

Los resultados de la investigación permiten observar la relevancia de la opinión de los viajeros en la preservación del patrimonio ferroviario, donde la memoria y su relación con la identidad, son centrales en la construcción de la territorialidad. En este sentido, tal como se afirmó en las discusiones teóricas, la preeminencia de la identidad es clave para el fomento de aquellos objetos icónicos de los lugares habitados, donde el ferrocarril aun cumple un papel protagónico en el desarrollo sociocultural de la región de estudio.

\section{Discusión}

Los estudios evaluativos, sean de cualquier índole, implican profundizar en el conocimiento y la percepción respecto a variables o categorías que se presentan como problemáticas, relacionadas directamente con el tránsito histórico y social de las personas. En este sentido, la presente investigación permitió develar la evaluación que hacen los habitantes de La Araucanía que utilizan el ferrocarril como dispositivo de transporte, más allá de lo particularmente relacionado con un objeto interurbano que permite el desplazamiento. Cabe destacar la alta vacancia respecto a los estudios nacionales que opten por un enfoque cuantitativo para observar fenómenos sociales vinculados a la valoración del ferrocarril, lo que implica que esta investigación aporte, desde sus limitaciones, una perspectiva innovadora en la comprensión del ferrocarril como dispositivo que no solo está ligado a la planificación de las ciudades y la movilidad de objetos y humanos.

Un primer elemento que queda en evidencia se relaciona con las transformaciones culturales y económicas del ferrocarril en el último tiempo (Guerrero, 2017). Ante ello, los resultados pesquisados permiten comprender que el servicio regional analizado, a pesar de su potencialidad, continúa en un estado de parsimonia respecto a su gradual amplitud de servicios pregonada por algunos gobiernos 
de turno, lo cual es altamente identificado por la muestra de la investigación, siendo operacionalizado a partir de los resultados exhibidos en las figuras 3 y 6 , como una realidad negativa que impacta en una evaluación regular y deficiente del servicio a nivel genérico.

Un segundo elemento reside en que, para los habitantes rurales y urbanos de la región que participaron de la investigación, el ferrocarril está íntimamente ligado con la historia familiar y la vida social de las ciudades que habitan, comprendiendo las ciudades como realidades complejas en permanente tensión y cambios (Gilabert, 2017). En este sentido, el planteamiento de Méndez y Cuellar (2017), vinculado con la interpretación de lo patrimonial, implica pensar el fuerte lazo afectivo e identitario entre los usuarios del ferrocarril y la memoria (Jelin, 2002), y su capacidad de organizar recuerdos y acciones respecto a la historia de vida. Los resultados analizados en la Tabla 3 dan cuenta de cómo los pasajeros relacionan el ferrocarril con su historia personal, donde la activación de la memoria conlleva deconstruir visiones estructuradas sobre un medio de transporte, haciendo de este un objeto central en la respectiva vida social de los participantes.

De esta forma, se asocia la valoración del servicio a la construcción de identidad en torno al patrimonio ferroviario, con el pasado y, por tanto, con la memoria de las comunidades. El ferrocarril es también la evidencia material de prácticas sociales y culturales del pasado: fue un motor en la construcción de historias locales, causa de la transformación de comunidades y de la dinamización de sus identidades, tras crear nuevos e inéditos espacios de sociabilidad. Por lo mismo, el ferrocarril es, al mismo tiempo, un vestigio histórico del pasado y un elemento aglutinador de memorias. Estas memorias, al igual que las identidades, no son ni convergentes ni homogéneas. Hay una importante diversidad de memorias, al menos tantas como sujetos sociales vinculados al ferrocarril.

Un tercer elemento está dado por el ferrocarril y su rol en la vida cotidiana y las prácticas sociales actuales y pasadas de muchas personas, adquiriendo una dimensión experiencial que, sin dudas, ha contribuido a generar un trasfondo simbólico y cultural que impacta en la identidad de los territorios.

El ferrocarril no es solo un medio de transporte con valor histórico y económico, sino también el símbolo de un tipo de sociabilidad que ha creado una realidad cultural inevitablemente asociada a procesos identitarios, símbolo al que los diversos sujetos le otorgan significados específicos. Esto queda reflejado en los resultados descritos en la Figura 7, donde diversas variables, con significados múltiples de lo material e inmaterial, implican la construcción de lo patrimonial desde la perspectiva de los usuarios del servicio.

Reflexionar sobre este tercer elemento, conlleva interrogar las normas sociales respecto a lo que debe considerarse patrimonial y aquello que no. Los filtros, propulsados desde las relaciones de poder (García Canclini, 1999), implican discutir sobre la vigencia residual del patrimonio industrial, donde aún persiste cierto nivel de vacuidad en la escena académica nacional respecto a la revitalización y preservación del patrimonio ferroviario y sus múltiples entradas analíticas desde lo material e inmaterial, dando cuenta de su potencialidad como bien cultural y social (Guzmán, Fernández, Basavilbaso y Bordoli, 2016).

Figura 7

Factores patrimoniales.

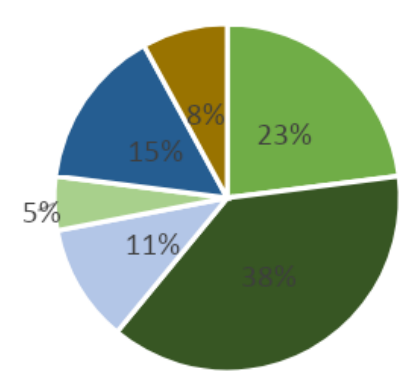

- Paisajes

- Estaciones - Ciudades

- Recuerdos

- Familia

- publicidad

Fuente: Elaboración propia.

\section{Conclusiones}

La hipótesis planteada en esta investigación se corrobora, debido a dos factores centrales. Por un lado, al reconocer 
la valoración del servicio, se logra profundizar en la calidad de vida de las personas desde las implicancias territoriales. No todos -y especialmente los habitantes de la ruralidad- tienen acceso de manera expedita a sus lugares de frecuentación, lo que permite que el ferrocarril continúe siendo significativo en la vida cotidiana de las personas. Por otro lado, al reconocer la relevancia del ferrocarril, pese a su disminuida cobertura geográfica, se reafirma la vigencia de este dispositivo de transporte, como un objeto que rebasa su papel en el desplazamiento, para así posicionarse en el desarrollo cultural (desde lo patrimonial y la memoria), económico (desde la historia y la política) y social (desde el compromiso con el territorio) de una región con alta desigualdad social y escaso reconocimiento intercultural.

Quedan aún abiertas las reflexiones sobre las disputas en torno al sentido patrimonial del ferrocarril VictoriaTemuco, en cuanto vehículo de memoria e identidad de sus usuarios. El estudio, más bien exploratorio, no tuvo por objetivo profundizar en las dimensiones de la conflictualidad de este patrimonio, aunque muestra que existe cierta diversidad de significados referidos a la valoración patrimonial, los que, no obstante, no son contradictorios.

En este sentido, se interpreta que el patrimonio ferroviario presenta una significación social compartida, pero, sobre todo, vivenciada de forma individual, cuestión que repercute en una baja conciencia patrimonial de los participantes, al menos en su dimensión colectiva. En este marco, la ausencia de una reivindicación activa de este patrimonio por parte de los usuarios podría responder a la poca relevancia patrimonial que se le da, en particular, al servicio ferroviario VictoriaTemuco desde la institucionalidad pública y la misma empresa. Probablemente, la falta de interés "oficial" por el patrimonio ferroviario, al menos en cuanto a este servicio en específico, se ha instalado como un "modelo" hegemónico relativo a la significación patrimonial del ferrocarril Victoria -Temuco, desincentivando el interés de los usuarios por activar este repertorio patrimonial y reivindicar su significación para la memoria e identidad propia. En efecto, se podría hablar de un proceso de patrimonialización de "baja intensidad" en el que las disputas de sentidos son más bien potenciales y no manifiestas.

Cabe destacar que los avances y restricciones de la presente investigación constituyen una invitación para continuar analizando la valoración del transporte ferroviario, como una vía que permite reconocer variables asociadas a la construcción patrimonial de lo ferroviario desde la experiencia y percepción humana. Por consiguiente, los hallazgos aquí presentados, abren más preguntas que respuestas, y con el objetivo de continuar con futuras investigaciones en esta línea, se plantean aristas relacionadas con, por ejemplo, el rol del ferrocarril en los espacios de vida actuales; la relación entre el ferrocarril y el entorno urbano y rural, considerando diferencias y similitudes respecto a la planificación de los territorios, y, la necesidad de incorporar a gran escala en las políticas públicas el patrimonio ferroviario, como muestra de la historia pasada, presente y futura de las ciudades del sur de Chile.

Finalmente, cada pequeño resultado de investigaciones que han contribuido y se han interesado en el análisis del ferrocarril, desde variadas perspectivas y en múltiples espacios académicos, constituyen piezas claves en la construcción de la identidad de aquellos territorios donde el ferrocarril de pasajeros continúa vigente en el territorio nacional, a pesar de su gradual estancamiento en lo que respecta a su funcionamiento. Conocer la valoración del servicio ferroviario implica contribuir en la detección de niveles de reconocimiento, revitalización y preservación del ferrocarril en la historia reciente de las ciudades chilenas.

\section{Referencias bibliográficas}

Alliende, M. P. (2017). La construcción de los Ferrocarriles en Chile 1850-1913. Revista Austral de Ciencias Sociales, (5), 143-161. https://doi.org/10.4206/rev.austral.cienc. soc.2001.n5-13 
Augé, M. (2009). El viajero subterráneo. Un etnólogo en el metro. Xalapa: Al fin liebre.

Cantar, N. (2019). Hacia una mirada dialéctica del patrimonio. Question, 1(61), 1-15. https://doi. org/10.24215/16696581e141

Ceniceros, B. y Ettinger, C. (2020). Paisaje urbano desde la frontera Juárez-El Paso. Mapeando manifestaciones de arte urbano desde el bordo. EURE (Santiago), 46(137), 181-201. http://dx.doi.org/10.4067/S0250-71612020000100181

Dormaels, M. (2011). Patrimonio, patrimonialización e identidad. Hacia una hermenéutica del patrimonio. Revista Herencia, 24(1 y 2), 7-14. https://revistas.ucr. ac.cr/index.php/herencia/article/view/1432

Dourton, A. (1994). Un régimen urbanístico en la América Hispana colonial: el trazado de damero durante los siglos XVI y XVII. Revista Historia, 28, 59-115. https:// repositorio.uc.cl/handle/11534/9531

Flores, J. (2012). La Araucanía y la construcción del sur de Chile, 1880-1950. Turismo y vías de transporte. Scripta Nova, 16. https://revistes.ub.edu/index.php/ScriptaNova/ article/view/14800

Fusté-Forné, F. (2018). Viajes en tren: el territorio y la comunicación de la identidad. Dixit, (28), 4-11. https:// dx.doi.org/10.22235/d.v0i28.1577

García, J. (1994). El hombre medieval como "homo viator": Peregrinos y Viajeros. IV Semana de Estudios Medievales, Nájera, del 2 a 6 de agosto de 1993.

García Canclini, N. (1999). Los usos sociales del patrimonio cultural. En Encarnación Aguilar C. (coord.), Patrimonio Etnológico. Nuevas perspectivas de estudio. Sevilla: Consejería de Cultura, Junta de Andalucía.

Gilabert, M. (2017). Experiencias en la intervención del patrimonio industrial. Estudio comparativo. Pasos. Revista de Turismo y Patrimonio Cultural, 15(2), 459-470. https://doi.org/10.25145/j.pasos.2017.15.029
Guajardo, G. (2007). Tecnología, Estado y ferrocarriles en Chile, 1850-1950. México: Fundación de los Ferrocarriles Españoles.

Guarda, G. (1978). Historia urbana del Reino de Chile. Santiago: Andrés Bello.

Guerrero, R. (2012). Patrimonio cultural mundial, territorio y construcción de ciudadanía. Construcción y apropiación social del patrimonio cultural de la ciudad de Valparaíso Chile. Scripta Nova, 16, 387-424. https://revistes.ub.edu/ index.php/ScriptaNova/article/view/3417/3678

Guerrero, R. (2017). Memorias, significados y olvidos en la construcción social del patrimonio ferroviario del Sur de Chile. Revista Austral de Ciencias Sociales, (33), 59-76. https://doi.org/10.4206/rev.austral.cienc. soc.2017.n33-04

Gutiérrez, A. (2012). ¿Qué es la movilidad? Elementos para (re)construir las definiciones básicas del campo del transporte. Bitácora, 261-74. https://doi.org/10.15446/ bitacora.v2n21.29076

Guzmán, A., Fernández, G., Basavilbaso, H. y Bordoli, M. (2016). Patrimônio industrial e turismo: análisis de potencialidade y propuesta de circuito de turismo industrial en Barracas, Buenos Aires, Argentina. Revista Brasileira de Pesquisa en Turismo, 10(1), 152-172. https:// dx.doi.org/10.7784/rbtur.v10i1.999

Instituto Nacional de Estadística. (2017). Censo 2017. Santiago: Autor.

Jelin, E. (2002). Los trabajos de la memoria. Madrid: Siglo Veintiuno Editores.

Méndez, R. y Cuéllar, D. (2017). Apuntes sobre la construcción del patrimonio ferroviario en España durante el siglo XX: identidad y museos. Oculum ensaios, 14(2), 275-292. https://dx.doi.org/10.24220/2318-0919v14n2a3895

Ministerio de Desarrollo Social. (2017). Encuesta de Caracterización Socioeconómica Nacional. Santiago: Autor. 
Musset, A. (2009). ¿Geohistoria o geoficción? Ciudades vulnerables y justicia espacial. Medellín: Editorial Universidad de Antioquia.

Núñez, A. (2010). La ciudad como sujeto: formas y procesos de su constitución moderna en Chile, siglos XVIII y XIX. Revista de Geografía Norte Grande, (46), 45-66. http:// dx.doi.org/10.4067/S0718-34022010000200003

Prats, L. (1997). Antropología y patrimonio. Barcelona: Ariel.

Riquelme, H. y Oyarce, F. (2019). Construcción de la historia reciente del ferrocarril de La Araucanía (Chile) desde la percepción de sus trabajadores. Revista Sophia Austral, (24), 5-24. http://dx.doi.org/10.4067/S0719$\underline{56052019000200005}$

Rodríguez, S. (1999). Patrimonio cultural y patrimonio antropológico. Revista de Dialectología y Tradiciones Populares, LIV(2), 107-123.

Rojas-Mix, M. (2002). La plaza mayor. San José: Editorial de la ciudad de Costa Rica.
Rojas, M. (2015). Dialéctica del patrimonio. Modernizaciones y cultura activa en disputa. México: Del Lirio / UAM.

Sagredo, R. (2010). El atlas de Gay. La representación de una nación. En Claudio Gay, Atlas de la Historia Física y Política de Chile (pp. IX-LXXVI). Santiago: Cámara Chilena de la Construcción, PUC de Chile, Bibl. Nacional de Chile.

Torres, C. (2013). Los riesgos por el "abandono cultural" del patrimonio industrial: maestranzas ferroviarias en Chile, Apuntes, 26(2), 52-67. https://revistas.javeriana. edu.co/index.php/revApuntesArq/article/view/10666

Valencia, M. (2017). Tensiones entre procesos de patrimonialización y modernización neoliberal. El caso de los paisajes culturales modernos: conjuntos habitacionales y barrios obreros en América Latina en el siglo XX. Revista de Urbanismo, (37), 3-16. http:// dx.doi.org/10.5354/0717-5051.2017.45198 